\title{
Adaptive Fuzzy Sliding Mode Control for the Probe Soft Landing on the Asteroids with Weak Gravitational Field
}

\author{
Yuanchun Li, ${ }^{1}$ He Wang, ${ }^{1}$ Bo Zhao, ${ }^{2}$ and Keping Liu ${ }^{1}$ \\ ${ }^{1}$ Department of Control Engineering, Changchun University of Technology, Changchun 130012, China \\ ${ }^{2}$ State Key Laboratory of Management and Control for Complex Systems, Institute of Automation, \\ Chinese Academy of Sciences, Beijing 100190, China \\ Correspondence should be addressed to Bo Zhao; zhaob09@mails.jlu.edu.cn and Keping Liu; liukeping@ccut.edu.cn
}

Received 27 January 2015; Revised 7 May 2015; Accepted 7 May 2015

Academic Editor: Francesco Franco

Copyright (C) 2015 Yuanchun Li et al. This is an open access article distributed under the Creative Commons Attribution License, which permits unrestricted use, distribution, and reproduction in any medium, provided the original work is properly cited.

\begin{abstract}
For the trajectory control of the probe soft landing on the asteroids with weak gravitational field, this paper presents a combined integral sliding mode control with an adaptive fuzzy logic system, named adaptive fuzzy sliding mode control (AFSMC) scheme. Considering the uncertainty of the orbit dynamics model in the small body fixed coordinate system, and the polyhedron modeling uncertainty in the gravitational potential, a fuzzy logic system is adopted to approximate the upper bound of the uncertainties. In addition, a robust control item is introduced to compensate for the approximation error of fuzzy logic system. The designed adaptive law and robust item make the closed-loop control stable and the tracking errors are convergent to zero. The controller not only guarantees the rapidity and accuracy of the desired trajectory tracking, but also enhances the robustness of the control system, improving the dynamic tracking performance for the probe soft landing on asteroids. Finally, the contrastive simulation results are presented to show the feasibility and effectiveness of the proposed control scheme.
\end{abstract}

\section{Introduction}

Soft landing on asteroids is one of the most complex tasks in deep space exploration. In the process of soft landing, the probe should not only achieve the most superior performance of the fuel in the decline stage, but also ensure the accuracy of soft landing. In order to guarantee the probe lands on asteroids safely and successfully, it is necessary to control and adjust the trajectory and velocity of the probe in real time.

Many scholars domestically and overseas have done a lot of researches specific to the trajectory control for the probe soft landing, which is the most important link to achieve the exploration tasks successfully. Yang et al. [1] proposed a fuzzy sliding mode control scheme for the probe to achieve the soft landing according to the nominal trajectory by the means of utilizing fuzzy rules to adjust the sliding control gain as well as adopting adaptive law to compensate for the system uncertainty. Aimed at the declining and landing control of the probe, Cui et al. [2] decomposed the landing control into the velocity control and deceleration control and then designed a proportional guidance law with a terminal condition and a deceleration control law. Liu et al. [3] presented a continuous control scheme based on PD and nonsingular terminal sliding mode for the probe descending and safe landing. However, in the simulations of the methods mentioned above, the sine or cosine functions with the same frequency were taken as the uncertainty and disturbance, whose demonstrated results cannot verify the effectiveness of these strategies when the uncertainty and disturbance were in the form of different frequency and complex signal. Taking this problem into account, Cui et al. [4] developed a soft landing autonomous impulse maneuver control method to deal with the parameter uncertainty of the weak gravitational field of small body, and the robustness of the landing control was greatly improved. Zhang et al. [5] proposed a probe autonomous navigation strategy to achieve the fast tracking by adopting a sliding mode variable structure control method. Carson et al. [6] utilized a robust sliding mode predictive control for the trajectory tracking of the small body proximity operations. Many of these methods considered 
the uncertainty and disturbance; however, the strict stability proofs were not given in detail theoretically; thus they were not enough and impractical for real applications $[7,8]$.

Due to the strong robustness and autonomy to the model uncertainty and the disturbance, fuzzy sliding mode control has been employed widely in the fields such as robot, spacecraft attitude control. Liu and Sun [9] designed an adaptive fuzzy terminal sliding mode control for second-order nonlinear system to guarantee the output error convergence to zero in finite time. Nekoukar and Erfanian [10] combined the continuous nonsingular terminal sliding mode with the adaptive learning algorithm and the fuzzy logic system to estimate the uncertain MIMO nonlinear system model and guaranteed the closed-loop system stability. Boubakir et al. [11] proposed a neural-fuzzy-sliding mode controller using the nonlinear sliding surface for the coupled tank system; the chattering phenomenon was weakened. Kuo et al. [12] designed an adaptive fuzzy sliding mode controller for the Lorenz system based on the output of the sliding mode controller, in which the adaptive technique and fuzzy logic rules were adopted to deduce the reaching law to ensure the state of the Lorenz system to reach the sliding mode surface. Besides, Wang et al. [13] proposed an optimal nonlinear control law based on a fuzzy neural network based on the optimum trajectory for lunar optimal trajectory tracking descent. $\mathrm{Xu}$ et al. [14] combined the fuzzy and variablestructure control to guide the terminal landing for lunar landing; it was more robust than some classical guidance laws derived from the linearized dynamics. But, in actual fact, the landing on an asteroid is much more complicated than that on moon, for the reason that the asteroids are always irregularly sharp and of uneven quality and uncertain rotary shaft, and so forth; thus these strategies should be improved to be feasibly applied to the probe soft landing on the asteroids.

In many previous works on the probe soft landing tasks, the asteroid was considered as a three-axis ellipsoid, whose gravitational potential is generally expressed as a secondorder spherical harmonic expansion [15-17]. However, this approximated model for the asteroid with weak gravitational field has many inaccuracies due to its irregular shape and uneven mass distribution. In this paper, the AFSMC scheme is designed to achieve the fast tracking of the desired trajectory and velocity based on a more precise gravitational polyhedron attraction model. Meanwhile, the integral sliding mode item is applied to eliminate the steady-state error. For the modeling uncertainty, external disturbance, and approximated error, an adaptive fuzzy item is proposed to overcome its upper bound, which improves the control accuracy. Finally, the robust control and adaptive law enables the system to achieve the close-loop system stability and guarantees the tracking error convergence to zero asymptotically, which eventually make the probe achieve a soft landing successfully. The main contributions of this work lie in that the AFSMC scheme has been carried out for the probe soft landing on the asteroids with weak gravitational field. Different from the other literatures that considered the asteroid as a three-axis ellipsoid, whose gravitational potential is generally expressed as a second-order spherical harmonic expansion, this paper approximates the asteroids'

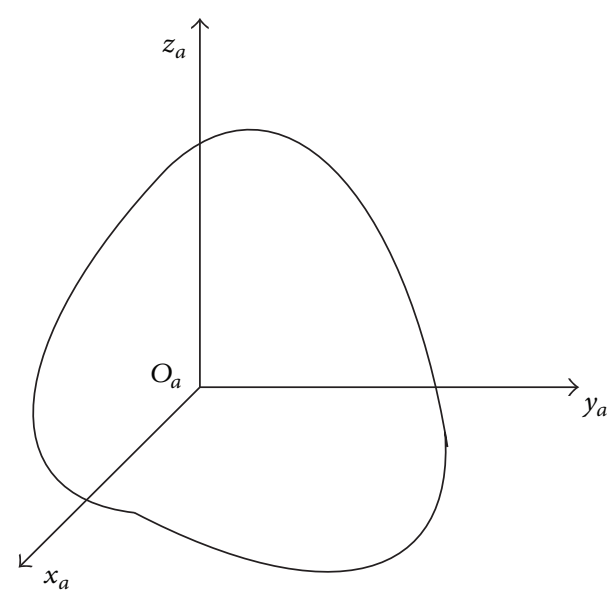

Figure 1: Coordinate system of the probe.

model as gravitational polyhedron attraction mode, which can bring about a more precise model. Meanwhile, the model uncertainty and disturbance are handled theoretically to guarantee the stability of the closed-loop system, which makes it more safe and feasible to be applied in practice.

\section{Dynamic Model}

2.1. Coordinate System and Gravitational Polyhedron Attraction. As shown in Figure 1, define the asteroid body-fixed coordinate system $O_{a} x_{a} y_{a} z_{a}$, where $O_{a}$ is the center of mass of the small body and $O_{a} x_{a}$ and $O_{a} z_{a}$ coincide with the minimum inertia axis and the spin axis of the asteroid, respectively. $O_{a} y_{a}, O_{a} x_{a}$, and $O_{a} z_{a}$ axes compose the righthanded coordinate system.

Here, a more precise gravitational attraction model than that established by spherical harmonic expansion is adopted. The three-dimensional solid body of the asteroid consists of a number of faces meeting at each vertex, and the connective topology can be also described as edges connecting the vertex pairs and the face pairs. With the assumptions as (1) the asteroid is a polyhedron and (2) the polyhedron's density is constant, the gravitational field can be generally modeled as [18]

$$
\begin{aligned}
U_{p}= & \frac{1}{2} G \sigma \sum_{e \in \text { edges }} \mathbf{v}_{e} \cdot \mathbf{E}_{e} \cdot \mathbf{v}_{e} \cdot L_{e} \\
& -\frac{1}{2} G \sigma \sum_{f \in \text { faces }} \mathbf{v}_{f} \cdot \mathbf{F}_{f} \cdot \mathbf{v}_{f} \cdot \psi_{f}
\end{aligned}
$$

where $G$ is the gravitational constant; $\sigma$ is the constant density of the asteroid; $\mathbf{v}_{e}$ is a vector from the field point to an arbitrary point on each edge; $\mathbf{v}_{f}$ is a vector from the field point to an arbitrary point on each face; $\mathbf{E}_{e}$ is a dyad defined in terms of the face and edge normal vectors associated with each edge; $\mathbf{F}_{f}$ is the outer product of face normal vectors; $L_{e}$ is a logarithmic term expressing the potential of a 1D straight wire; and $\psi_{f}$ is the solid angle subtended by a face when viewed from the field point. 
And the polyhedron attraction which is obtained by the time derivative of (1) can be expressed as

$$
\nabla U=-G \sigma \sum_{e \in \text { edges }} \mathbf{E}_{e} \cdot \mathbf{r}_{e} \cdot L_{e}+G \sigma \sum_{f \in \text { faces }} \mathbf{F}_{f} \cdot \mathbf{r}_{f} \cdot \omega_{f} .
$$

2.2. Probe Dynamic Model. The dynamic equations of the probe in the fixed-body coordinate system are given by [19]

$$
\begin{aligned}
\ddot{x}-2 w \dot{y}-w^{2} x & =g_{x}+U_{x}+D_{x}, \\
\ddot{y}+2 w \dot{x}-w^{2} y & =g_{y}+U_{y}+D_{y}, \\
\ddot{z} & =g_{z}+U_{z}+D_{z},
\end{aligned}
$$

where $w$ is the spin rate of the asteroid, $x, y$, and $z$ are the components of the probe position vector in the body-fixed coordinate system, $\left[\begin{array}{lll}g_{x} & g_{y} & g_{z}\end{array}\right\rfloor=\left\lfloor\begin{array}{llll}\nabla U_{x} & \nabla U_{y} & \nabla U_{z}\end{array}\right\rfloor$ is the polyhedron attraction vector in three axes, $\left|\begin{array}{llll}U_{x} & U_{y} & U_{z}\end{array}\right|$ is the relative control acceleration vector in three axes, and $D_{x}$, $D_{y}$, and $D_{z}$ denote the sum of the modeling uncertainties in each direction, respectively.

\section{Controller Design and Stability Analysis}

3.1. Desired Trajectory Design. Nominal profile is designed to make the probe reach the intended landing site within the time $\tau$ and satisfy all the landing constraints. In order to land on asteroid safely, the vertical velocity must be small enough so as not to cause the probe rollover or damage.

We employ a cubic polynomial method to plan the falling trajectory [20], which can be described as

$$
x_{d}(t)=x_{0}+x_{1} t+x_{2} t^{2}+x_{3} t^{3}
$$

with the initial states and final states as follows:

$$
\begin{aligned}
& x_{d}(0)=x_{0}, \\
& \dot{x}_{d}(0)=\dot{x}_{0}, \\
& x_{d}(\tau)=x_{t}, \\
& \dot{x}_{d}(\tau)=\dot{x}_{t} .
\end{aligned}
$$

Then the desired trajectory, velocity, and acceleration of $x$-axis can be written as

$$
\begin{aligned}
x_{d}(t)= & x_{0}+\dot{x}_{0} t-\frac{3 x_{0}+2 \dot{x}_{0} \tau-3 x_{t}+2 \dot{x}_{t} \tau}{\tau^{2}} t^{2} \\
& +\frac{2 x_{0}+\dot{x}_{0}-2 x_{t}+\dot{x}_{t} \tau}{\tau^{3}} t^{3}, \\
v_{d}(t)= & \dot{x}_{0}-2 \frac{3 x_{0}+2 \dot{x}_{0} \tau-3 x_{t}+2 \dot{x}_{t} \tau}{\tau^{2}} t \\
& +3 \frac{2 x_{0}+\dot{x}_{0}-2 x_{t}+\dot{x}_{t} \tau}{\tau^{3}} t^{2}, \\
a_{d}(t)= & -2 \frac{3 x_{0}+2 \dot{x}_{0} \tau-3 x_{t}+2 \dot{x}_{t} \tau}{\tau^{2}} \\
& +6 \frac{2 x_{0}+\dot{x}_{0}-2 x_{t}+\dot{x}_{t} \tau}{\tau^{3}} t .
\end{aligned}
$$

Similarly, the desired trajectories, velocities, and accelerations of $y$-, $z$-axes can be determined.

Define the tracking error as

$$
\underline{\mathbf{e}}=\mathbf{r}-\mathbf{r}_{\mathbf{d}}
$$

where $\underline{\mathbf{e}}=\left[\begin{array}{llllll}e_{1} & e_{2} & e_{3} & \dot{e}_{1} & \dot{e}_{2} & \dot{e}_{3}\end{array}\right]$ and $\mathbf{r}_{\mathbf{d}}=$ $\left[\begin{array}{lllllll}x_{d} & y_{d} & z_{d} & \dot{x}_{d} & \dot{y}_{d} & \dot{z}_{d}\end{array}\right]$.

Considering the trajectory control problem for the soft landing of the probe, and with constraint (5) held, the control objective of this paper is to design an adaptive fuzzy sliding mode control law $U$ to make the actual trajectory $\mathbf{r}$ track the desired trajectory $\mathbf{r}_{\mathbf{d}}$. In other words, the designed control law $U$ can guarantee that

(1) the closed-loop system is stable, and all signals are uniformly bounded;

(2) tracking error $e_{i}(i=1,2,3)$ converges to the equilibrium point.

3.2. Description of Fuzzy Logic System. Assume that the fuzzy system is a map from $V \subseteq R^{n}$ to $R, V=V_{1} \times \cdots \times V_{n}, V_{i} \subset R$, $i=1,2, \ldots, n$; then the $k$ th typical fuzzy rules represented in IF-THEN form can be expressed as

$$
\begin{aligned}
& \mathbf{R}^{(k)}: \text { if } p_{1} \text { is } A_{1}^{k} \text { and } \ldots \text { and } p_{n} \text { is } A_{n}^{k} \text { then } q \text { is } G^{k} \\
& (k=1,2, \ldots, N),
\end{aligned}
$$

where $A_{i}^{k} \in\left\{A_{i}^{1}, \ldots, A_{i}^{n_{i}}\right\}(i=1,2,3)$ are the fuzzy sets with memberships $\mu_{A_{i}^{k}}, y$ is a linguistic variable, respectively, and $N=\prod_{i=1}^{n} n_{i}$ is the number of fuzzy rules.

Through the fuzzification and center average defuzzifier, the output of fuzzy logic system can be expressed as

$$
q=\theta^{T} \xi(p)
$$

where $\theta^{T}=\left[\begin{array}{llll}\theta^{1} & \theta^{2} & \ldots & \theta^{N}\end{array}\right]^{T}$ is a vector set of parameters and $\xi^{T}=\left[\begin{array}{llll}\xi_{1} & \xi_{2} & \cdots & \xi_{N}\end{array}\right]$ is a set of fuzzy basic functions, which is defined as [21]

$$
\xi_{k}(p)=\frac{\prod_{i=1}^{n} \mu_{A_{i}^{k}}\left(p_{i}\right)}{\sum_{k=1}^{N}\left(\prod_{i=1}^{n} \mu_{A_{i}^{k}}\left(p_{i}\right)\right)} \quad k=1, \ldots, N
$$

3.3. Adaptive Fuzzy Integral Sliding Mode Controller Design. Sliding mode control has strong robustness for the system with the existence of uncertainty and disturbance [22] and can obtain high control accuracy. Classical sliding mode control is very sensitive to the interference in the approaching movement stage, which is a period of time when the system has not yet reached the sliding mode surface [23]. This phenomenon will influence the control performance. In order to overcome this disadvantage, an integral sliding mode surface is defined as

$$
s_{i}=\dot{e}_{i}+\alpha_{i} e_{i}+k_{i} \int_{0}^{t} e_{i}(\tau) d \tau,
$$


where $i=1,2,3$, the surface vector of sliding mode is $S=$ $\left[\begin{array}{lll}s_{1} & s_{2} & s_{3}\end{array}\right]^{T}$, and the parameters $\alpha_{i}, k_{i}$ can make all the roots of the equation $h_{i}\left(s_{i}\right)=\ddot{s}_{i}+\alpha_{i} \dot{s}_{i}+k_{i}$ be located in the left half-plane of the complex plane.

The classical sliding mode control (CSMC) law with integral sliding surface is defined by

$$
\begin{aligned}
& U_{x}=-\left(w^{2} x+2 w \dot{y}+g_{x}\right)+\vartheta_{x}-u_{s w 1}, \\
& U_{y}=-\left(w^{2} y-2 w \dot{x}+g_{y}\right)+\vartheta_{y}-u_{s w 2}, \\
& U_{z}=-g_{z}+\vartheta_{z}-u_{s w 3},
\end{aligned}
$$

where

$$
u_{s w}=\left(\begin{array}{l}
\eta_{\Delta 1} \operatorname{sgn}\left(s_{1}\right) \\
\eta_{\Delta 2} \operatorname{sgn}\left(s_{2}\right) \\
\eta_{\Delta 3} \operatorname{sgn}\left(s_{3}\right)
\end{array}\right)
$$

with $\eta_{\Delta i}>\left|d_{i}\right|$ being a positive constant.

Considering the system model uncertainty and the disturbance, a compensation item $\vartheta$ is designed as (14) based on (11), which ensures that the state can be achieved and kept in the sliding surface:

$$
\vartheta=\ddot{Y}_{d}-\Lambda_{1} \dot{e}-\Lambda_{0} e,
$$

where $\ddot{Y}_{d}=\left[\begin{array}{lll}\ddot{x}_{d} & \ddot{y}_{d} & \ddot{z}_{d}\end{array}\right]^{T}, \Lambda_{1}=\operatorname{diag}\left(\alpha_{11}, \alpha_{21}, \alpha_{31}\right), \Lambda_{0}=$ $\operatorname{diag}\left(k_{1}, k_{2}, k_{3}\right)$, and $\dot{e}=\left[\begin{array}{lll}\dot{e}_{x} & \dot{e}_{y} & \dot{e}_{z}\end{array}\right]^{T}$.

Assumption 1 . The system uncertainty $D$ is norm bounded with $\|D\| \leq \Gamma(r)$, where $\Gamma(r) \geq 0$ is an unknown function.

Ideal fuzzy system (15) is adopted to approximate the unknown linear function $\Gamma\left(r \mid \theta_{\Gamma}\right)$ :

$$
\Gamma\left(r \mid \theta_{\Gamma}\right)=\theta_{\Gamma}^{T} \xi(r),
$$

where $\theta_{\Gamma}^{T}=\left[\begin{array}{llll}\theta_{\Gamma}^{1} & \theta_{\Gamma}^{2} & \cdots & \theta_{\Gamma}^{N}\end{array}\right]^{T}$ is the member function and the form of the fuzzy vector $\xi(r)$ is given as (10). And its estimation is

$$
\widehat{\Gamma}\left(r \mid \widehat{\theta}_{\Gamma}\right)=\widehat{\theta}_{\Gamma}^{T} \widehat{\xi}(r) .
$$

To eliminate the fuzzy approximation errors, the robust control items $u_{1}=\left[\begin{array}{lll}u_{11} & u_{12} & u_{13}\end{array}\right], u_{2}=\left[\begin{array}{lll}u_{21} & u_{22} & u_{23}\end{array}\right]$ are introduced to the control law, which can be rewritten as [24]

$$
\begin{aligned}
& U_{x}=-\left(w^{2} x+2 w \dot{y}+g_{x}\right)+\vartheta_{x}-\operatorname{sat}\left(s_{1}\right)+u_{11}+u_{21}, \\
& U_{y}=-\left(w^{2} y-2 w \dot{x}+g_{y}\right)+\vartheta_{y}-\operatorname{sat}\left(s_{2}\right)+u_{12}+u_{22}, \\
& U_{z}=-g_{z}+\vartheta_{z}-\operatorname{sat}\left(s_{3}\right)+u_{13}+u_{23},
\end{aligned}
$$

where

$$
\begin{aligned}
& u_{1}=-\frac{S}{\left\|S^{T}\right\|} \widehat{\Gamma}\left(r \mid \widehat{\theta}_{\Gamma}\right), \\
& u_{2}=-\frac{S}{\left\|S^{T}\right\|} \widehat{w}_{\Gamma} .
\end{aligned}
$$

The parameters of the variable can be updated by the following adaptive laws:

$$
\begin{aligned}
& \dot{\theta}_{\Gamma}=\gamma_{\Gamma} \hat{\xi}(r)\left\|S^{T}\right\|, \\
& \dot{\widehat{w}}_{\Gamma}=\gamma_{w}\left\|S^{T}\right\|,
\end{aligned}
$$

where $\gamma_{\Gamma}>0, \gamma_{w}>0$ are some suitable constants representing the adaptive gains.

\subsection{Stability Analysis}

Theorem 2. Considering the probe dynamic model (3) and Assumption 1, with the integral sliding mode control law designed as (17) and adaptive update laws as (19), the closedloop system is stable and all the variables will asymptotically converge to zero.

Proof. Define the optimal approximation parameter as

$$
\theta_{\Gamma}^{*}=\underset{\theta_{\Gamma} \in \Omega_{\Gamma}}{\arg \min }\left(\sup _{r \in \Omega_{r}}\left|\Gamma_{i}\left(r \mid \theta_{\Gamma}\right)-\widehat{\Gamma}\left(r \mid \widehat{\theta}_{\Gamma}\right)\right|\right),
$$

where $\theta_{\Gamma}$ is continuous on the compact set $\Omega_{\Gamma}$.

Then the parameter errors can be defined as

$$
\widetilde{\theta}_{\Gamma}=\theta_{\Gamma}^{*}-\theta_{\Gamma}
$$

The approximation error of fuzzy logic system can be obtained as

$$
\Gamma(r)-\widehat{\Gamma}\left(r \mid \theta_{\Gamma}^{*}\right)=\widetilde{\theta}_{\Gamma} \widehat{\xi}(r)+\widehat{\theta}_{\Gamma} \widetilde{\xi}_{(r)}+\widetilde{\theta}_{\Gamma} \widetilde{\xi}(r) .
$$

Assume that

$$
\|\omega\|=\left\|\widehat{\theta}_{\Gamma} \widetilde{\xi}(r)+\widetilde{\theta}_{\Gamma} \tilde{\xi}(r)\right\| \leq w_{\Gamma},
$$

where $w_{\Gamma}$ is a positive constant, whose approximate error can be defined as

$$
\widetilde{w}_{\Gamma}=w_{\Gamma}-\widehat{w}_{\Gamma} .
$$

From (3) and (17), we can get

$$
\dot{S}=u_{1}+u_{2}-\operatorname{sat}(s)+D .
$$

Define the Lyapunov function as

$$
V=\frac{1}{2} S^{T} S+\frac{1}{2 \gamma_{\Gamma}}\left(\widetilde{\theta}_{\Gamma}^{T} \widetilde{\theta}_{\Gamma}\right)+\frac{1}{2 \gamma_{w 1}} \widetilde{w}_{\Gamma 1}^{2} .
$$




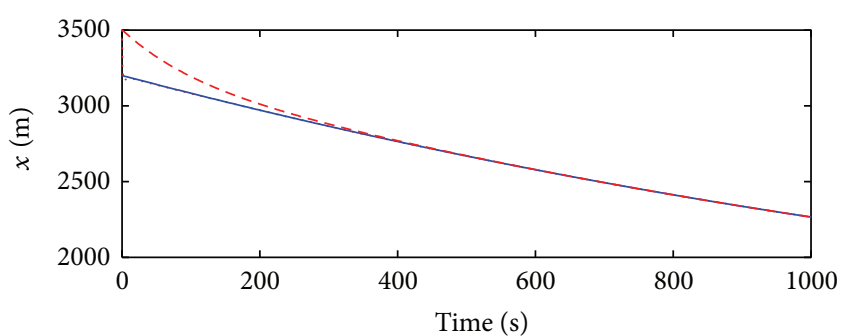

.... Actual position with AFSMC
- Desired position of $x$-axis
- - Actual position with CSMC

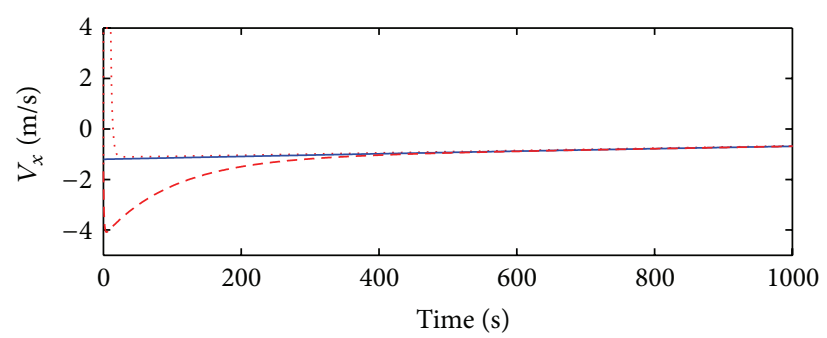

… Actual velocity with AFSMC

- Desired velocity of $x$-axis

- - - Actual velocity with CSMC

FIgURE 2: Position and velocity tracking curves of the $x$-axis.

Differentiating (26) with respect to time, with consideration of assumption and the adaptive laws (19), yields

$$
\begin{aligned}
\dot{V}= & S^{T} \dot{S}-\frac{1}{\gamma_{\Gamma}} \widetilde{\theta}_{\Gamma}^{T} \dot{\hat{\theta}}_{\Gamma}-\frac{1}{\gamma_{w}} \widetilde{w}_{\Gamma}^{T} \dot{\hat{w}}_{\Gamma} \\
= & S^{T}\left(-\operatorname{sgn}(S)+u_{1}+u_{2}+D\right)-\frac{1}{\gamma_{\Gamma}} \widetilde{\theta}_{\Gamma}^{T} \dot{\hat{\theta}}_{\Gamma} \\
& -\frac{1}{\gamma_{w}} \widetilde{w}_{\Gamma}^{T} \dot{\hat{w}}_{\Gamma} \\
\leq & -\left\|S^{T}\right\|-\left\|S^{T}\right\| \widehat{\Gamma}\left(r \mid \widehat{\theta}_{\Gamma}\right)-\left\|S^{T}\right\| \widehat{w}_{\Gamma} \\
& +\left\|S^{T}\right\| \Gamma\left(r \mid \theta_{\Gamma}\right)-\frac{1}{\gamma_{\Gamma}} \widetilde{\theta}_{\Gamma}^{T} \dot{\hat{\theta}}_{\Gamma}-\frac{1}{\gamma_{w}} \widetilde{w}_{\Gamma}^{T} \dot{\hat{w}}_{\Gamma} \\
\leq & -\left\|S^{T}\right\|+\left\|S^{T}\right\|\left(\widetilde{\theta}_{\Gamma}^{T} \widehat{\xi}\right)+\left\|S^{T}\right\|\left(w_{\Gamma}-\widehat{w}_{\Gamma}\right)-\frac{1}{\gamma_{\Gamma}} \widetilde{\theta}_{\Gamma}^{T} \dot{\hat{\theta}}_{\Gamma} \\
& -\frac{1}{\gamma_{w}} \widetilde{w}_{\Gamma}^{T} \dot{\hat{w}}_{\Gamma} \\
= & -\left\|S^{T}\right\|+\widetilde{\theta}_{\Gamma}^{T}\left(\left\|S^{T}\right\| \widehat{\xi}-\frac{1}{\gamma_{\Gamma}} \dot{\hat{\theta}}_{\Gamma}\right) \\
& +\widetilde{w}_{\Gamma}^{T}\left(\left\|S^{T}\right\|-\frac{1}{\gamma_{w}} \dot{\hat{w}}_{\Gamma}\right) \leq 0,
\end{aligned}
$$

where $\operatorname{sgn}(S)=\left[\begin{array}{lll}\operatorname{sgn}\left(s_{1}\right) & \operatorname{sgn}\left(s_{2}\right) & \operatorname{sgn}\left(s_{3}\right)\end{array}\right]^{T}$ and $|S|=\left(\left|s_{1}\right|+\right.$ $\left.\left|s_{2}\right|+\left|s_{3}\right|\right)$. Thus, the time derivative of Lyapunov function (26) is negative definition which guarantees the closed-loop system convergence to the equilibrium.

Note. To reduce the chattering phenomenon of control signals, the saturated function (28) is instead of the sign function [25]:

$$
\operatorname{sat}(\cdot)= \begin{cases}\frac{s}{\Phi} & |s| \leq \Phi \\ \operatorname{sgn}(s) & |s|>\Phi\end{cases}
$$

where $\Phi>0$ is the width of saturation function in the sliding mode surface.
TABLE 1: Landing simulation parameters.

\begin{tabular}{lcc}
\hline Name & Value & Unit \\
\hline Desired initial position & {$\left[\begin{array}{ccc}3200 & 1300 & 9000\end{array}\right]$} & $\mathrm{m}$ \\
Desired initial velocity & {$\left[\begin{array}{lll}-1.2 & 0.2 & -1\end{array}\right]$} & $\mathrm{m} / \mathrm{s}$ \\
Landing position & {$\left[\begin{array}{cccc}2837 & 928.1 & 5708\end{array}\right]$} & $\mathrm{m}$ \\
\hline
\end{tabular}

\section{Simulation Results}

Taking the Eros 433 as the target asteroid [26] with the spin angular velocity $w=3.31 \times 10^{-4} \mathrm{~m} / \mathrm{s}$, the constraint values of the probe at initial points and landing site are given in Table 1, as has been worked out in this section. We adopt the probe model (3) in the fixed coordinate system, the cubic polynomial desired trajectory (6), the CSMC scheme (12), and the proposed AFSMC scheme (17), respectively, for the comparable simulation, in order to illustrate the superiority of the proposed control scheme.

Introduce the model uncertainty and disturbance as

$$
\begin{aligned}
& D_{x}=0.1 \sin (0.005 t) \cdot g_{x}, \\
& D_{y}=0.2 \sin (0.005 t) \cdot g_{y}, \\
& D_{z}=-0.2 \cos (0.005 t) \cdot g_{z} .
\end{aligned}
$$

The falling time is $8000 \mathrm{~s}$; other simulation parameters are set as $\Lambda_{1}=\left[\begin{array}{lll}1 & 1 & 1\end{array}\right], \Lambda_{0}=\left[\begin{array}{lll}0.1 & 0.1 & 0.1\end{array}\right], \Phi=0.4$, and $\gamma_{\Gamma}=\gamma_{w}=0.1 \times 10^{-5}$. To observe the results more clearly, the simulation time is set as 8000 seconds in the 3D descending tracking trajectory, 100 seconds in control acceleration, and 1000 seconds in the others. The simulation results are shown in Figures 2-7.

Figures 2-4 illustrate the contrast position and velocity tracking curves in three directions, respectively, and Figure 5 shows their tracking error. We can observe that the probe can track the desired trajectories and the desired velocities in three axes within only 20 seconds with the proposed AFSMC scheme; it shows that not only a stable state, but also a priority convergent rate and smaller tracking errors than those of CSMC (12) with the same control parameters in [27] can be achieved. Figure 6 presents the 3D descending trajectory tracking curves, from where one can observe that the probe 


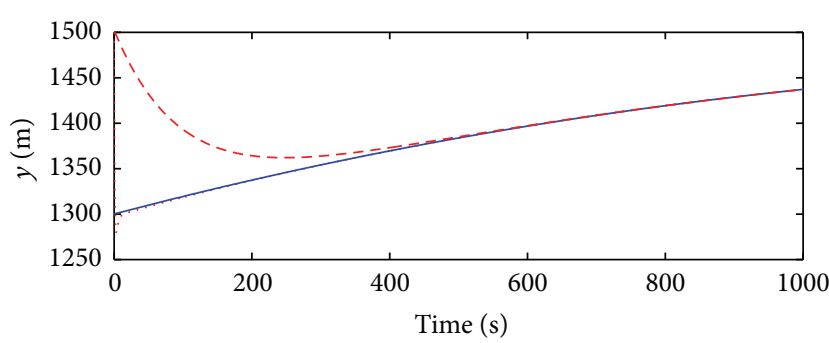

Actual position with AFSMC

- Desired position of $y$-axis - - - Actual position with CSMC

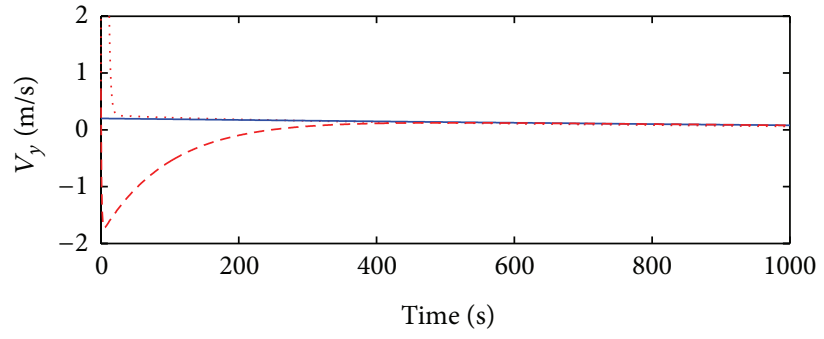

Actual velocity with AFSMC

- Desired velocity of $y$-axis

- - - Actual velocity with CSMC

FIGURE 3: Position and velocity tracking curves of the $y$-axis.

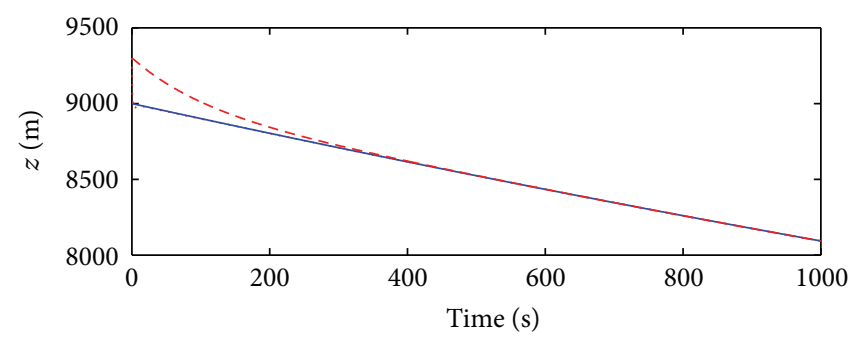

Actual position with AFSMC

Desired position of $z$-axis Actual position with CSMC

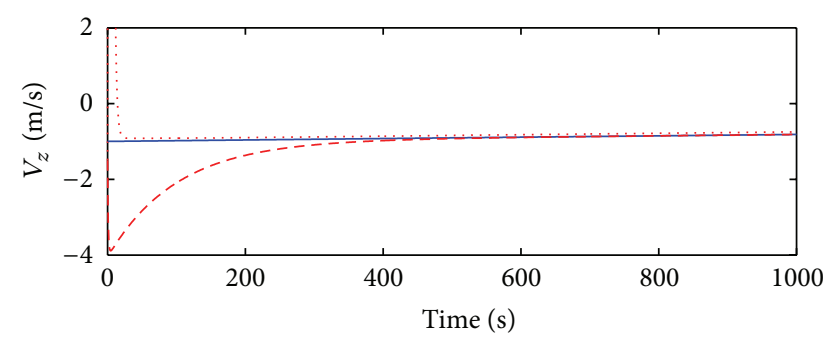

Actual velocity with AFSMC

- Desired velocity of $z$-axis

- - - Actual velocity of CSMC

FIgURE 4: Position and velocity tracking curves of the $z$-axis.

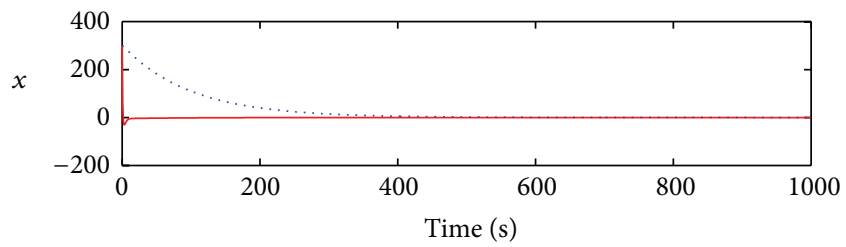

Time (s)

- Position error with AFSMC

Position error with CSMC

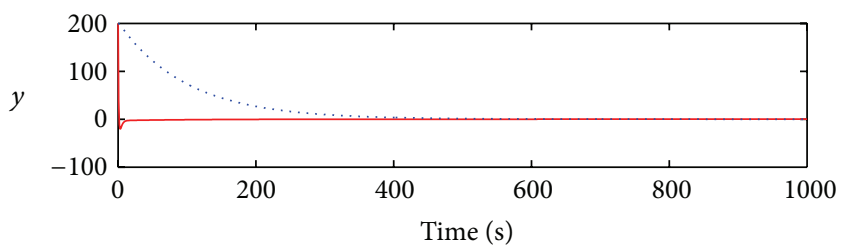

- Position error with AFSMC

..... Position error with CSMC

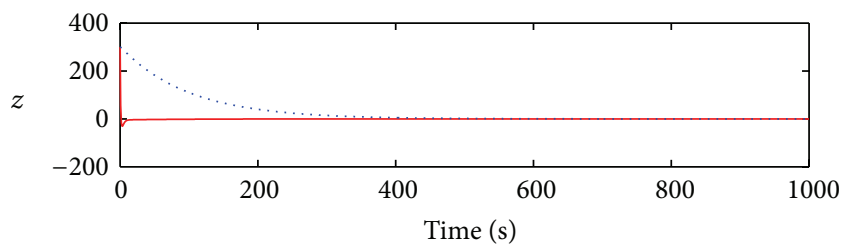

— Position error with AFSMC

Position error with CSMC

FIgURE 5: Position error curves.

can land on the asteroid surface softly. In general, the time cost can be greatly improved by the proposed AFSMC, though with some control acceleration chattering existence (shown in Figure 7). Therefore, the proposed scheme is more feasible and effective in practice to achieve our main goal.

\section{Conclusions}

Considering the existence of uncertainties and external disturbance of the probe when it lands on a weak gravitational field asteroid, an AFSMC scheme is proposed in 


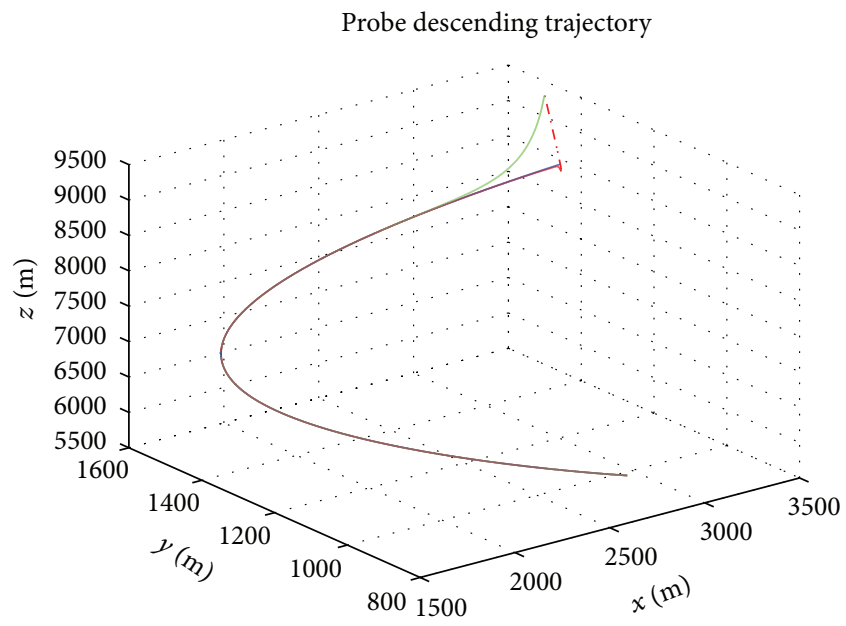

... Actual descending trajectory with AFSMC

— Desired descending trajectory

— Actual descending trajectory with CSMC

FIGURE 6: Descending trajectory of the probe.
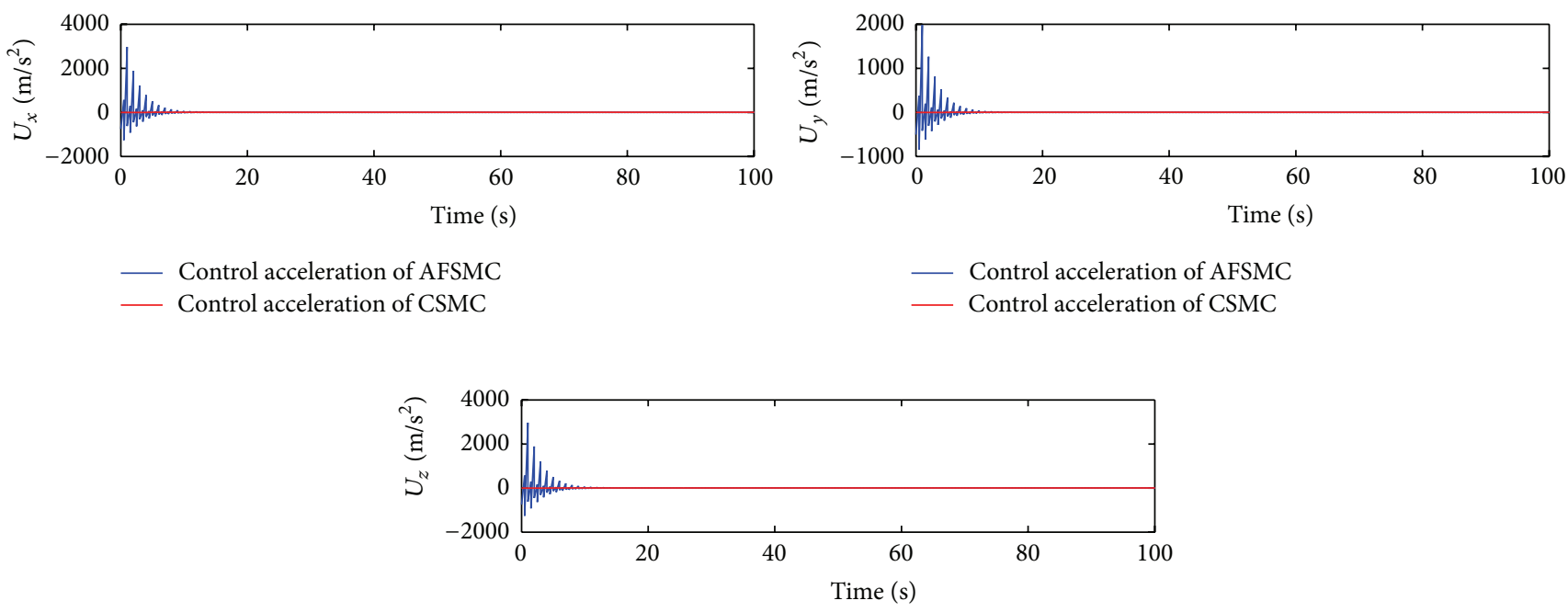

- Control acceleration of AFSMC

_ Control acceleration of CSMC

FIgURE 7: Control acceleration.

this paper. The fuzzy logic, whose fuzzy weight is updated adaptively, is applied to approximate the upper bound of the modeling uncertainties to reduce the effects on the control precision and enhance the control accuracy. Furthermore, the integral sliding mode control method is utilized to track the desired trajectory and restrain steady-state error along with enhancing the dynamic performance of the system. Finally, adaptive laws are adopted to update the control parameters in real time and robust item is introduced to eliminate the fuzzy approximation errors, which makes the system stable. Simulation verifies that the control scheme is effective and feasible to achieve the soft landing of the probe.

\section{Conflict of Interests}

The authors declare that there is no conflict of interests regarding the publication of this paper.

\section{Acknowledgment}

This work is supported by the National Basic Research Program of China (973 Program) under Grant 2012CB720000.

\section{References}

[1] Y.-N. Yang, J. Wu, and W. Zheng, "Trajectory tracking for an autonomous airship using fuzzy adaptive sliding mode control," 
Journal of Zhejiang University SCIENCE C, vol. 13, no. 7, pp. 534543, 2012.

[2] H.-T. Cui, X.-Y. Shi, and P.-Y. Cui, "Guidance and control law for soft landing asteroid," Jounal of Flight Dynamics, vol. 20, pp. 35-38, 2002.

[3] K. Liu, J. Zeng, B. Zhao, and Y. Li, "Continuous control for probe landing based on Terminal sliding mode," Journal of Beijing University of Aeronautics and Astronautics, vol. 40, no. 10, pp. 1323-1328, 2014.

[4] P. Cui, S. Zhu, and H. Cui, "Autonomous impulse maneuver control method for soft landing," Journal of Astronautics, vol. 29, pp. 121-126, 2008.

[5] Z. Zhang, W. Wang, L. Li et al., "Robust sliding mode guidance and control for soft landing on small bodies," Journal of the Franklin Institute, vol. 349, no. 2, pp. 493-509, 2012.

[6] J. M. Carson III, B. Açikmeşe, R. M. Murray, and D. G. MacMynowski, "Robust model predictive control with a safety mode: applied to small-body proximity operations," in Proceedings of the AIAA Guidance, Navigation and Control Conference and Exhibit, Honolulu, Hawaii, USA, August 2008.

[7] C. Liang and Y. Li, "Attitude analysis and robust adaptive backstepping sliding mode control of spacecrafts orbiting irregular asteroids," Mathematical Problems in Engineering, vol. 2014, Article ID 367163, 15 pages, 2014.

[8] R. Furfaro, "Hovering in asteroid dynamical environments using higher-order sliding control," Journal of Guidance, Control, and Dynamics, vol. 38, no. 2, pp. 263-279, 2015.

[9] J. Liu and F. Sun, "Chattering free adaptive fuzzy terminal sliding mode control for second order nonlinear system," Journal of Control Theory and Applications, vol. 4, no. 4, pp. 385391, 2006.

[10] V. Nekoukar and A. Erfanian, "Adaptive fuzzy terminal sliding mode control for a class of MIMO uncertain nonlinear systems," Fuzzy Sets and Systems, vol. 179, pp. 34-49, 2011.

[11] A. Boubakir, F. Boudjema, and S. Labiod, "A neuro-fuzzysliding mode controller using nonlinear sliding surface applied to the coupled tanks system," International Journal of Automation and Computing, vol. 6, no. 1, pp. 72-80, 2009.

[12] C.-L. Kuo, N.-S. Pai, and H.-T. Yau, "Adaptive fuzzy sliding mode controller design for Lorenz system," in Proceedings of the International Workshop on Chaos-Fractals Theories and Applications, pp. 122-125, November 2009.

[13] D.-Y. Wang, G.-D. Qiao, and T.-S. Li, "FNN guidance control for lunar optimal trajectory tracking descent," Journal of Astronautics, vol. 28, no. 5, pp. 1149-1170, 2007.

[14] M. Xu, Y. He, Y. Liang, and X. Ding, "Terminal guidance strategy for a hybrid thrust-tether lunar landing scheme," Advances in Space Research, vol. 55, no. 9, pp. 2280-2292, 2015.

[15] G. Romain and B. Jean-Pierre, "Ellipsoidal harmonic expansions of the gravitational potential: theory and application," Celestial Mechanics and Dynamical Astronomy, vol. 79, no. 4, pp. 235-275, 2001.

[16] Z. Zhenjiang, C. Hutao, and R. Gaofeng, "Modeling for the graviation potential environment of an irregular-shaped asteroid and the spherical harmonic coefficient estimation," Spacecraft Environment Engineering, vol. 27, pp. 383-388, 2010.

[17] E. Herrera-Sucarrat, P. L. Palmer, and R. M. Roberts, "Modeling the gravitational potential of a nonspherical asteroid," Journal of Guidance, Control, and Dynamics, vol. 36, no. 3, pp. 790-798, 2013.
[18] R. A. Werner and D. J. Scheeres, "Exterior gravitation of a polyhedron derived and compared with harmonic and mascon gravitation representations of asteroid 4769 Castalia," Celestial Mechanics and Dynamical Astronomy, vol. 65, no. 3, pp. 313344, 1997.

[19] S. Li, H.-T. Cui, and P.-Y. Cui, "Autonomous GNC technology for landing on small bodies," The Journal of Astronautics, vol. 27, no. 1, pp. 21-26, 2006.

[20] L. Shuang and C. Pingyuan, "Variable structure with slidingmode control for landing on asteroids," Journal of Astronautics, vol. 26, no. 6, pp. 808-812, 2005.

[21] W.-S. Lin and C.-S. Chen, "Robust adaptive sliding mode control using fuzzy modelling for a class of uncertain MIMO nonlinear systems," IEE Proceedings: Control Theory and Applications, vol. 149, no. 3, pp. 193-202, 2002.

[22] V. I. Utkin and H.-C. Chang, "Sliding mode control on electromechanical systems," Mathematical Problems in Engineering, vol. 8, no. 4-5, pp. 451-473, 2002.

[23] F. Castaños and L. Fridman, "Analysis and design of integral sliding manifolds for systems with unmatched perturbations," IEEE Transactions on Automatic Control, vol. 51, no. 5, pp. 853$858,2006$.

[24] S. Aloui, O. Pagès, A. El Hajjaji, A. Chaari, and Y. Koubaa, "Improved fuzzy sliding mode control for a class of MIMO nonlinear uncertain and perturbed systems," Applied Soft Computing Journal, vol. 11, no. 1, pp. 820-826, 2011.

[25] P. Kachroo and M. Tomizuka, "Chattering reduction and error convergence in the sliding-mode control of a class of nonlinear systems," IEEE Transactions on Automatic Control, vol. 41, no. 7, pp. 1063-1068, 1996.

[26] Z. Zhang, H. Cui, and G. Ren, "Modeling for the gravitation potential environment of an irregular-shaped asteroid and the spherical harmonic coefficient estimation," Spacecraft Environment Engineering, vol. 7, pp. 383-388, 2010.

[27] G. Lingli and L. Yuanchun, "The motion decomposition of power descent section of soft landing on small bodies based on sliding mode and PD control algorithm," in Proceedings of the 4th International Conference on Intelligent Control and Information Processing, (ICICIP '13), pp. 318-322, June 2013. 